\title{
Persuasive and engaging design of a Smartphone App for cycle commuting
}

\author{
Yun-Maw Cheng ${ }^{*}$ and Chao-Lung Lee
}

\footnotetext{
* Correspondence: kevin@ttu.edu.tw The Graduate Institute of Design Science \& Department of Computer Science and Engineering, Tatung University, Taipei, Taiwan
}

\begin{abstract}
This article presents results from a pilot study addressed to investigate the design of behavior change intervention using smartphones for cycle commuting. In this specific case, a smartphone app, BikeTogether, was developed to encourage and support its users to cycle home with each other over the Internet. The app employs the metaphor of a bicycle flashlight to represent closeness, leading, and following between two sides. The cycling performance can also be recorded so the users can track how they are doing over time. 10 participants were instructed and randomly paired to take a two-phases test ride on different routes. Results indicated that the app can help create the sense of being with each other while cycling and promote not only accompanied but competing ride. In addition, the outcome of the desirability towards the app implies a higher chance it will lead to a behavior change. This provides a new way of seeing, being and knowing solitude, a state that we commit to remain encouraged.
\end{abstract}

Keywords: Copresence; Persuasive computing; Cycling app

\section{Introduction}

Cycling home after work is an example of sustainable transportation that accomplishes the commute and exercise with only one effort. However, the change of this kind is consequences of everyday decisions that assemble over time. Previous research has identified social influences and supports play a critical role in encouraging and retaining the change $[1,2]$. Can the pervasiveness and technological capabilities of smartphones be designed to enable the change and if so, how to leverage the materialities of smartphones to embody the social effect on motivating people for cycle commuting? Along with this idea, there are questions to be answered. What data are collected? How are the data turned into appropriate information and presentation, which is focused and meaningful enough for potential cycle commuters to understand and help them to make choices to hang on to the change?

Mounting a smartphone on the handlebar to track performance and share it to challenge friends is surge in popularity of cycling. The sharing is considered a key in creating a sense of togetherness [3,4]. Also, cycling home after work reminds us the importance of lighting. Feeling isolated is what augments the decreasing of the motivation. Lighting can create atmosphere and mood that makes us feel guided and accompanied [5,6]. As Fogg suggests, flashing lights is one of the form factors that can motivate people and prompt immediate action [7]. Utilizing the screen of a smartphone as the lighting source to

(c) 2015 Cheng and Lee. This is an Open Access article distributed under the terms of the Creative Commons Attribution License (http://creativecommons.org/licenses/by/4.0), which permits unrestricted use, distribution, and reproduction in any medium, provided the original work is properly credited. 
convey the exertion information in an ambient, unobtrusive, and intuitive manner could have the potential to embody social comparison to the commuters. To evaluate this experience, a series of studies were conducted to realize how those who new to cycle commuting interpreted and responded to the design.

\section{BikeTogether}

The goal of BikeTogether is to motivate people who just start cycle commuting to do it more by providing a tangible approach in creating a remote sense of togetherness. It exploits dynamic patterns of light, color and intensity, on the screen of a smartphone as sensory cues to define the relationship between the cycle-commuters' perception of their cycling activities. In order to have a better comprehension on how it works, an interaction walkthrough is shown below:

1. The users simply grab their bicycles, mount their smartphones on the handlebars, and turn on the app. They then come to the selection screen, which is a flashlight with an on/off button as the only one major user interface element. The button is a circular menu with four features, "Double", "Solo", "History", and "Setting". The users can circle along the selection and press the on/off icon to confirm the selection.

2. When the app runs up for the first time, the user enters information such as identity and password, to create an account. This is all done by going to "Setting" in the circular menu.

3. When "Double" is selected, the outer edge of the button starts flashing in white and this indicates that the system is attempting to pair two sides of the users whoever are online. If it becomes solid white, this indicates that the connection is successfully established and the system starts to facilitate the exchange of distance data between the users since they are paired. Otherwise, the process is again repeated.

4. Also, the users can compete with their previous best times and efforts by selecting "Solo". The connection with your virtual self is made instantly once the button is pressed.

5. The flashlight illuminates in different colors. Red, green, and orange lights denote following, leading, and closeness between the paired users in terms of the distance the users have ridden. The intensity is directly proportional to the level of acceleration and the flickering means the current positional state is about to change.

6. When the on/off button is pressed second time by either side of the users, the pair is ended immediately and a review of the current results is then brought up. The user can check distance, average speed, time (duration and date), and paired partner's ID of the cycling. A route map is shown on the screen as well. The map displays the leading, closeness, and following distance, represented by green, orange, and red respectively.

7. The users can browse their cycling records by selecting "History" on the selection screen at any time. For a more elaborate look at any individual cycling activity, click the bar representing that cycling to review the route map and other details (Figures 1 and 2). 


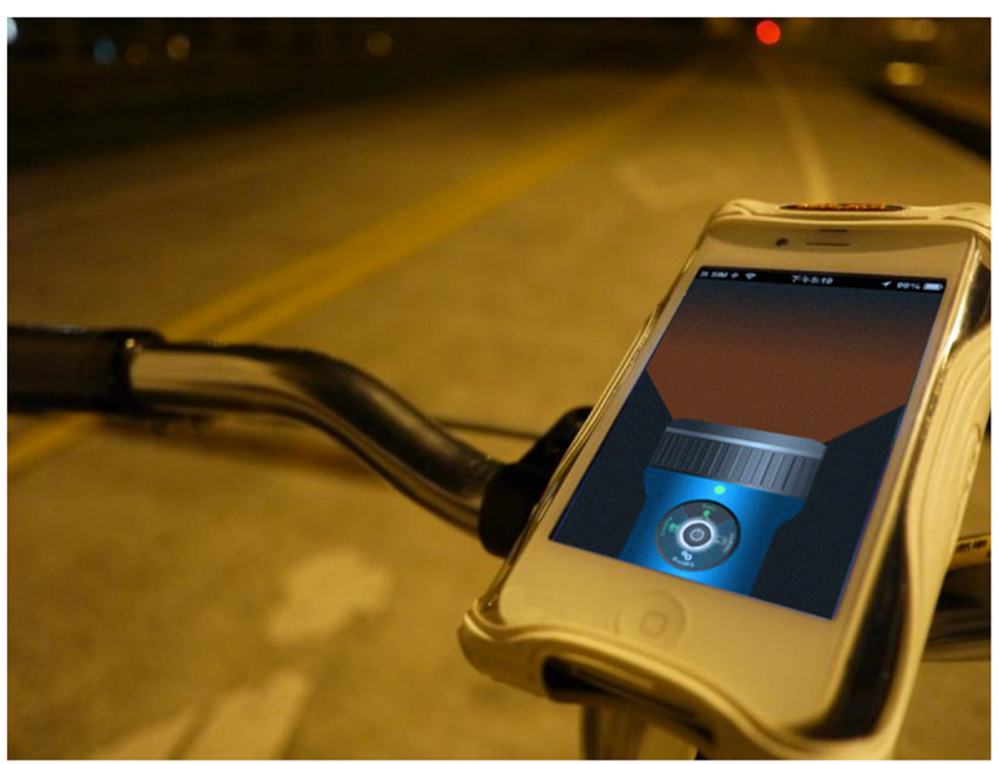

Figure 1 BikeTogether in the field.

\section{Evaluation}

In order to understand if BikeTogether can effectively convey information to people engaged in a cycling activity via the screen light from a smartphone mounted on the handlebar, and whether this can influence their attitude towards the transit of this kind, a two-phase experiment was designed and carried out with real users in the Dajia Riverside bicycle path, which is one of the busiest paths for cycle commuting in Taipei City. The participants consist of 10 undergraduate and postgraduate students at Tatung University ( 1 females, 9 males, $\mathrm{M}=23.1$ years, $\mathrm{SD}=1.14$ ). The majority of the participants (90\%) use cycling as a recreation activity, and half of them prefer cycling with a companion. On the day prior to the scheduled experiment, participants received a notification via Facebook Messenger to remind and confirm the appointment. Also, they were all notified to wear comfortable clothing.

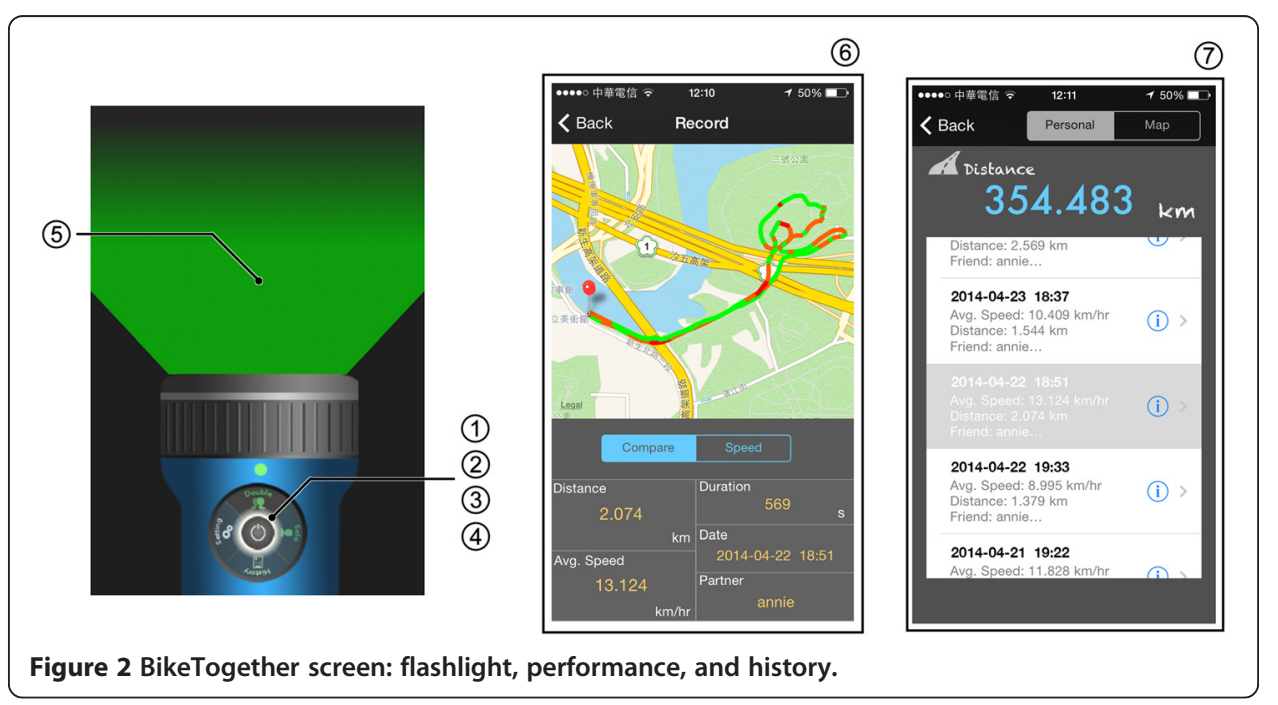


Two bicycles both with a smartphone mounted on the handlebars and BikeTogether preinstalled were prepared for the evaluation. The participants were then gathered, randomly split into 5 groups of two, and told to take a 10 minutes open cycle, which means they can ride on their own pace, on two different routes, one group at a time. As to how their cycling behavior is modeled, the mode of cycling is considered to be accelerating, decelerating, and constant speed in this evaluation. The cycling behavior of each participant therefore can be represented as an acceleration-time sequence within a certain time frame that meets the reality of cycling circumstances. In this case, a time frame of 5 seconds was chosen. These data were collected and calculated by the app ran in stealth mode without any GUI so the participants did not know they were being traced. After a 30 minutes rest, the participants were gathered once again and a brief instruction regarding the information coding of the app was given. They were then took another 10 minutes cycling with the app ran in normal mode, one group at a time. The data were again collected. Table 1 shows the rate of acceleration and deceleration in both study phases. The specified threshold for count is $\pm 2 \mathrm{~m} / \mathrm{sec}^{2}$.

A Wilcoxon signed-rank test showed a statistically significant difference before and after participants were aware of BikeTogether $(\mathrm{W}=10, \mathrm{P} \leq 0.05)$. Also, to better understand how the app effected on the participants, their relative position to each other in terms of the real-time cycling distance in the second phase was summarized and an interview was performed. All the participants reported the increase in their activity level and considered that it was mainly due to the more aware of their personal activity as well as the comparison with remote partner's provided by the app. Group 4 said that it started like a subtle competition and later it became more like a group ride. Group 1 and 2 expressed that, at first, they attempted to attack off the front and later figure out the different physical ability in between, then slow down to their moderate pace. A common group cycling formation was seen in Group 3 and 5. They commented that once in leading position they would lower their speed in order for the other peer to catch up.

In order to discover more about whether BikeTogether can engender positive effect that the users would use it frequently and longer, Production Reaction Cards was used to capture the participants' feelings and study their emotional involvement with BikeTogether [8]. These cards are composed of $60 \%$ of positive words and $40 \%$ of negative and neutral words. The participants were asked to select as many words as they wanted. The most selected word was fun (72\%), followed by entertaining (70\%), exciting (64\%) and motivating (51\%). All the participants uttered that the total cycling distance directly provides them the feeling of achievement. Also, they considered the acceleration and deceleration is the messages, which engage them in a form of conversation and enable them to

Table 1 The counts of acceleration and deceleration in each phase

\begin{tabular}{llllll}
\hline & \multicolumn{2}{l}{ BikeTogether (Stealth) } & & \multicolumn{2}{l}{ BikeTogether } \\
\cline { 2 - 3 } & Member A & Member B & & Member A & Member B \\
\hline Group 1 & 2 & 7 & 88 & 57 \\
Group 2 & 5 & 7 & 32 & 52 \\
Group 3 & 76 & 60 & 54 & 100 \\
Group 4 & 74 & 41 & 57 & 11 \\
Group 5 & 37 & 33 & 96 & 39 \\
\hline
\end{tabular}


freely express to each other in the comprehension that they might never meet again. This comprehension intrigues the thought of who and where the peers really are to maintain the surprising freshness. This makes us have confidence in that BikeTogether can help further enhance the cycling experience and has potential to nudge people who just start cycle commuting to keep up the change.

\section{Conclusion and future works}

The findings from this research indicate that smartphone apps can be designed to create a sense of togetherness between two cycle-commuters while cycling using the screen light. This leads to positive behavioral effects. However, the initial results that have raised a host of further questions:

- Will increased feelings of presence increase motivation in the long term so that the health and fitness goals will be achieved?

- Can the feedback be used to sustain the sense of presence in the longer term and will users find ways to appropriate the feedback mechanisms in ways that the designers never intended?

- Will the use of these complementary feedback mechanisms increase the feeling of presence without increasing the risks through distraction and ideally reducing the risks of cycling in large groups on busy roads?

The future plans include conducting a long-term in-the-wild observation based on the above questions and performing studies into factors that influence the users to take BikeTogether and use it more frequently. The factors, in turn, will be the evaluation framework to further extend the development boundary.

Received: 15 October 2014 Accepted: 11 March 2015

Published online: 28 March 2015

References

1. Kanning M, Schlicht W (2010) Be Active and Become Happy: An Ecological Momentary Assessment of Physical Activity and Mood. Journal of Sport and Exercise Psychology 32:253-261

2. Massung E, Coyle D, Cater KF, Jay M, Preist C (2013) Using crowdsourcing to support pro-environmental community activism. Paper presented at the SIGCHI Conference on Human Factors in Computing Systems, Paris, France

3. Zhao S (2003) Toward a Taxonomy of Copresence. Presence: Teleoperators and Virtual Environments 12(5):445-455

4. Bardzell J, Bardzell S, Zhang G, Pace T (2014) The lonely raccoon at the ball: designing for intimacy, sociability, and selfhood. Paper presented at the SIGCHI Conference on Human Factors in Computing Systems, Toronto, Ontario, Canada

5. Harrison C, Horstman J, Hsieh G, Hudson S (2012) Unlocking the expressivity of point lights. Paper presented at the SIGCHI Conference on Human Factors in Computing Systems, Austin, Texas, USA

6. Schmeiduch M, Marques A C, Frantzis K (2012) \#CPHSIGNALS, Copenhagen Institute of Interaction Design (CIID). Available via http://ciid.dk/education/portfolio/idp12/courses/systems-layers/projects/cphsignals/. Accessed 15 Oct 2014

7. Fogg BJ (2002) Persuasive Technology: Using Computers to Change What We Think and Do. Morgan Kaufmann Publishers, San Francisco, CA, USA

8. Benedek J, Miner T (2002) Measuring Desirability: New methods for evaluating desirability in a usability lab setting. Paper presented at Usability Professionals Association, Orlando, FL, USA 